Proceedings of the 43rd "Jaszowiec", International School and Conference on the Physics of Semiconductors, Wisła 2014

\title{
Fabrication of SbSI Photonic Crystals
}

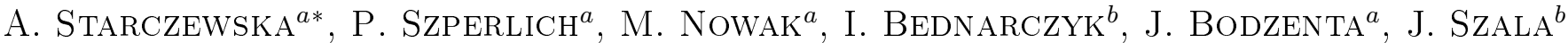 \\ ${ }^{a}$ Institute of Physics - Center for Science and Education, Silesian University of Technology, \\ Bolesława Krzywoustego 2, 44-100 Gliwice, Poland \\ ${ }^{b}$ Department of Materials Science, Silesian University of Technology, Z. Krasińskiego 8, 40-019 Katowice, Poland
}

\begin{abstract}
Semiconducting ferroelectric antimony sulfoiodide (SbSI) photonic crystals were fabricated. The $\mathrm{SiO}_{2}$ nanospheres were synthesized and gravity sedimented to obtain opal matrices. These opals were infiltrated with melted SbSI and etched in HF acid to produce inverted SbSI opals.
\end{abstract}

DOI: $10.12693 /$ APhysPolA.126.1118

PACS: 42.70.Qs, 77.84.--s, 78.40.Fy

\section{Introduction}

Photonic crystals (PCs) with periodically modulated dielectric constant have gathered great importance in recent years [1]. They possess a band gap for electromagnetic waves similar to that for electrons in crystalline semiconductors. Value of this gap strongly depends on geometrical parameters of the structure as well as on complex refractive indices of the applied materials. It is highly promising to use materials with refractive indices that can be tuned with external conditions, e.g. electric field or temperature. In this work antimony sulfoiodide (SbSI) was used for the construction of photonic crystal. SbSI is a ferroelectric semiconductor with relatively high refractive index strongly dependent on temperature [2] and external electric field, especially near its Curie point $\left(T_{\mathrm{C}}=292(1) \mathrm{K}\right)[3]$. Therefore, SbSI is potentially very useful in fabrication of tunable photonic crystals.

We applied method based on colloidal crystal templates that is relatively simple and efficient for fabrication of photonic crystals [4]. The basic idea is to infiltrate the spaces between the synthetic $\mathrm{SiO}_{2}$ spheres of the template with melted SbSI and then to remove the template by chemical etching to obtain inverted SbSI opals. The morphology of the $\mathrm{SiO}_{2}$ opals, $\mathrm{SiO}_{2}$ opals infiltrated with SbSI and inverted SbSI opals were investigated by scanning electron microscopy (SEM) and atomic force microscopy (AFM). The chemical composition of the samples was analysed using energy dispersion spectroscopy (EDS).

\section{Experiment}

Monodisperse silica spheres with diameter of several hundred nanometers range were prepared using procedure described in [5]. In this procedure the hydrolysis and condensation of tetraethylorthosilicate (TEOS) $\left[\mathrm{Si}(\mathrm{OR})_{4}\right.$ with $\left.\mathrm{R}=\mathrm{C}_{2} \mathrm{H}_{5}\right]$ proceeds in a mixture of alcohol

* corresponding author; e-mail: anna.starczewska@polsl.pl and water, with ammonia used as a catalyst. The $99 \%$ TEOS (purchased from Aldrich Chemical Co.) as well as ethanol (99.8\%) and 25\% ammonia (purchased from POCh, Gliwice) were used as starting materials. TEOS was added into the solution of ethanol, water and ammonia under stirring in constant temperature. The amounts of TEOS, ammonia, water and ethanol were chosen in order to reach final diameters of $\mathrm{SiO}_{2}$ particle in the several hundred nanometer range. The mixture was stirred for $2.5 \mathrm{~h}$ with a magnetic stirrer. Subsequently, the silica suspensions were centrifuged at $4000 \mathrm{rpm}$ for $30 \mathrm{~min}$ and washed with ethanol. The centrifuging/washing procedure was repeated six times and the final product was ultrasonically dispersed and stored in ethanol. Diameter of the synthesized $\mathrm{SiO}_{2}$ particles was determined from electron micrographs and optical investigations as it was described in [6].

Opals with thickness less than $0.5 \mathrm{~mm}$ were fabricated from the $\mathrm{SiO}_{2}$ particles using gravity sedimentation method $[5,7]$. The opals were sintered at temperature of $100{ }^{\circ} \mathrm{C}$ for $3 \mathrm{~h}$ to give them higher stability. Infiltration of the obtained $\mathrm{SiO}_{2}$ matrix with SbSI was performed in termisil ampoules and horizontal furnace. In typical process $0.100 \mathrm{~g} \mathrm{SiO}_{2}$ matrix and $0.500 \mathrm{~g}$ of SbSI single crystals were put into ampoules of length $150 \mathrm{~mm}$ and diameter $15 \mathrm{~mm}$. After evacuations $\left(p=10^{-3} \mathrm{mbar}\right)$ the ampoules were sealed and placed in furnace. In order to maintain the same temperature in every point of ampoule it was wrapped with a sheet of aluminum. The typical time of keeping $\mathrm{SiO}_{2}$ matrix in melted SbSI (in temperature $763 \mathrm{~K}$ ) was $96 \mathrm{~h}$. Then the ampoules were cooled with $5 \mathrm{~K} / \mathrm{min}$ rate. Opals infiltrated with SbSI were sonicated a few times in ethanol to remove single crystals from the surfaces.

To obtain SbSI inverted opals the $\mathrm{SbSI}-\mathrm{SiO}_{2}$ opal composite was immersed in $10 \%$ aqueous $\mathrm{HF}$ solution. The etching process of silica template was carried out at room temperature for $2 \mathrm{~min}$. Then the sample was washed with deionized water.

The chemical composition of the prepared samples was analysed using scanning electron microscope Hitachi S4200 with Thermo Scientific EDS. The morphology of 
the structures was characterized using Hitachi S-4200 and SEM STEM HITACHI HD-2300A microscopes, and PSIA XE-70 AFM working in non-contact mode. The BudgetSensors Tap300Al cantilevers (resonance frequency $300 \mathrm{kHz}$, force constant $40 \mathrm{~N} / \mathrm{m}$ ) were used. Acquired images were processed using image processing software (Gwyddion(R) software) to correct sample inclination and distortions caused by $z$-scanning stage. No other corrections in images were made.

\section{Results and discussion}

Figures 1 and 2 present the typical SEM micrographs of the top surface of bare $\mathrm{SiO}_{2}$ opal and inverted SbSI opal. One recognizes that the synthesized silica parti-

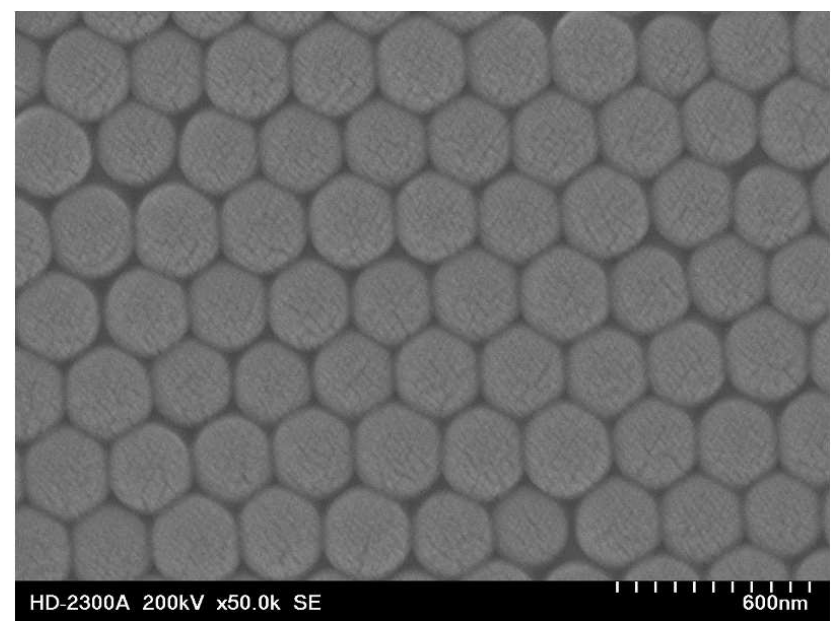

Fig. 1. Typical SEM micrographs of bare $\mathrm{SiO}_{2}$ opal used as a template for SbSI photonic crystal (diameter of the silica particles equals $286(18) \mathrm{nm}$ ).

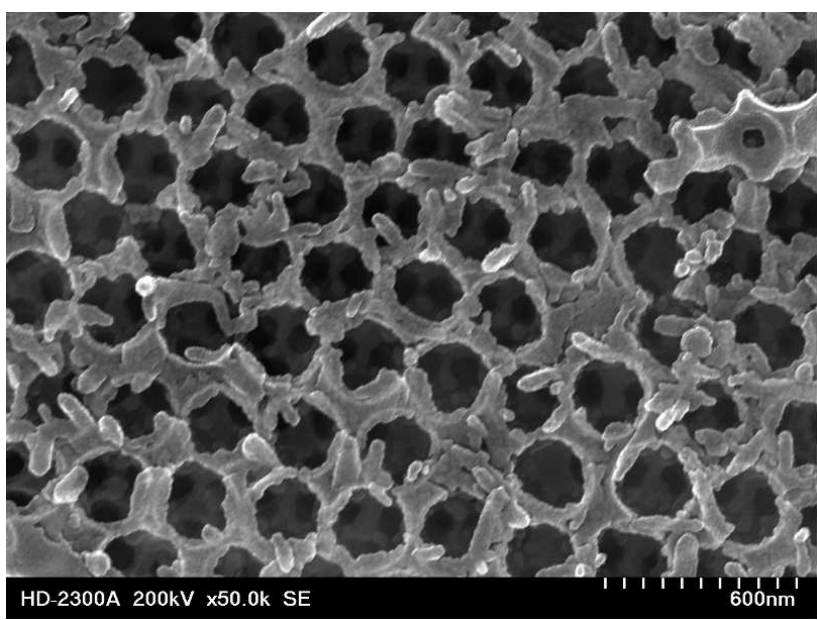

Fig. 2. Typical SEM micrographs of SbSI inverted opal (diameter of the air spheres fabricated after etching of silica particles equals $275(14) \mathrm{nm}$ ).

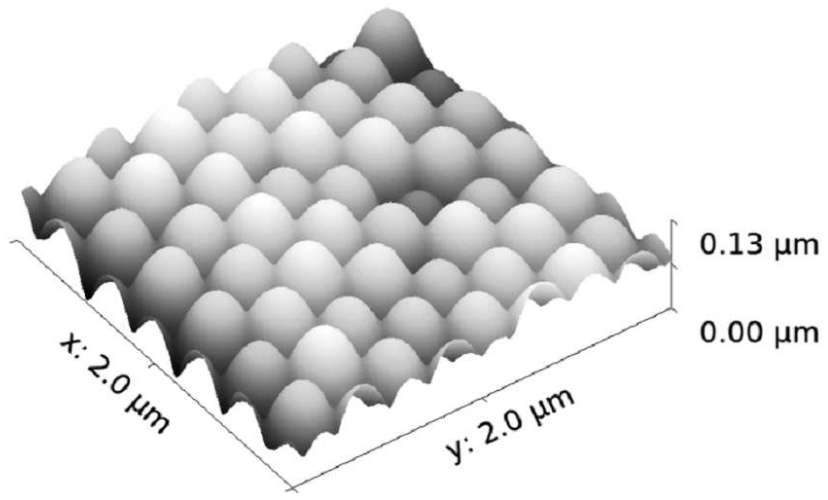

Fig. 3. AFM 3D image of $\mathrm{SiO}_{2}$ opal used as a template for SbSI photonic crystal (diameter of the silica particles equals $348(20) \mathrm{nm})$.

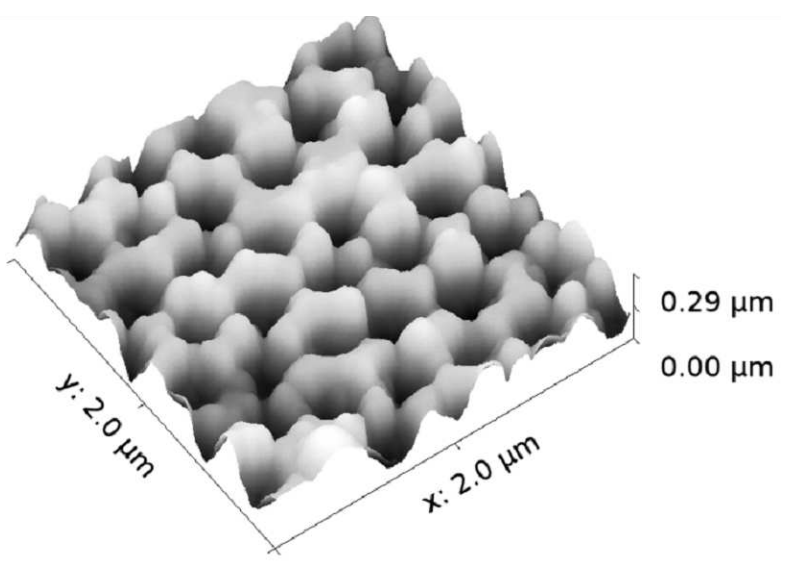

Fig. 4. AFM 3D image of inverted SbSI opal (diameter of the air spheres fabricated after etching of silica particles equals $348(20) \mathrm{nm}$ ).

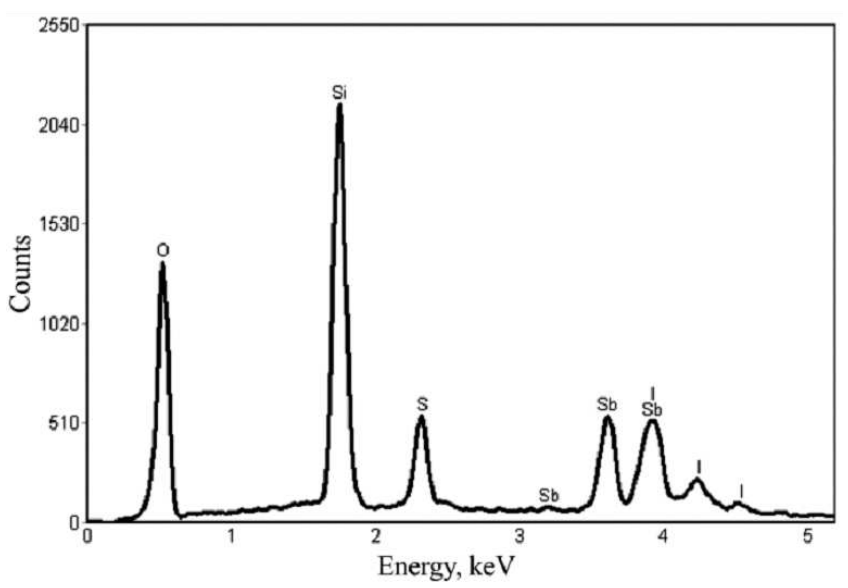

Fig. 5. EDS spectrum of the surface of $\mathrm{SiO}_{2}$ opal infiltrated with SbSI. 
cles are spherical and monodispersed. AFM images presented in Figs. 3 and 4 confirm the conclusions derived from SEM investigations. Diameters of the silica particles presented in various images are different due to differences in the ammonia concentrations used during each of the synthesis. Generally, it can be seen that the sample surfaces are parallel to the (111) plane of the opal. The characteristic $\mathrm{SiO}_{2}$ spheres of opal are clearly identified on the template surface (Fig. 1). The SEM images confirmed that the opal voids were filled with SbSI material (Fig. 2) The EDS analysis performed on $\mathrm{SiO}_{2}$ opals infiltrated with SbSI showed characteristic peaks for oxygen, silicon, antimony, sulfur and iodide (Fig. 5). An elemental atomic ratio of 0.38:0.30:0.32 for $\mathrm{Sb}, \mathrm{S}$ and I averaged over the sample surface was observed. These values are a little different from the appropriate for a stoichiometric SbSI. Authors of [8-10] established that material of such chemical composition is Sb-S-I glass. The XRD investigations of the $\mathrm{SiO}_{2}$ opals infiltrated with SbSI proved the existence of SbSI single crystals in amorphous media. Dimensions of SbSI crystal inclusions and the ratio of their volume to the volume of the final, inverted SbSI photonic crystals will be investigated in the future.

\section{Conclusions}

In summary, we described a simple route for producing SbSI photonic crystals. It is convenient, fast, mild, and efficient. The main optical properties of these photonic crystals were investigated by reflectance spectroscopy for wavelengths from $280 \mathrm{~nm}$ to $1000 \mathrm{~nm}$ [11]. These measurements exhibit Bragg's peaks connected with photonic band gap that is tunable in position and width by varying the medium filling the opal [11]. However, taking into account the fact that SbSI being a semiconductor ferroelectric has an unusually large number of interesting properties (among them there are the pyroelectric, pyro-optic, piezoelectric, electromechanical, electrooptic and other nonlinear optical effects), one should expect novel applications of SbSI inverted opals. Investigations of these applications are in progress.

\section{Acknowledgments}

This work was partially supported by the NCN (Poland) under Contract No. NN507250140.

\section{References}

[1] J.D. Joannopoulos, S.G. Johnson, J.N. Winn, R.D. Meade, Photonic Crystals: Molding the Flow of Light, Princeton University Press, Princeton 2008.

[2] M. Nowak, P. Szperlich, A. Kidawa, M. Kępińska, P. Gorczycki, B. Kauch, Proc. SPIE 5136, 172 (2003).

[3] P. Szperlich, M. Nowak, Ł. Bober, J. Szala, D. Stróż, Ultrason. Sonochem. 16, 398 (2009).

[4] F.Marlow, Muldarisnur, P.Sharifi, R. Brinkmann, C. Mendive, Angew. Chem. Int. Ed. 48, 6212 (2009).

[5] M. Kępińska, A. Starczewska, J. Szala, Opt. Mater. 36,932 (2014).

[6] A. Starczewska, J. Szala, M. Kepińska, M. Nowak, K. Mistewicz, M. Sozańska, Solid State Phenom. $\mathbf{1 9 7}, 119$ (2013).

[7] M. Kępińska, A. Starczewska, I. Bednarczyk, J. Szala, P. Szperlich, K. Mistewicz, Mater. Lett. 130, 17 (2014).

[8] I.D. Turyanitsa, B.M. Koperles, Izv. Akad. Nauk SSSR Neorganich. Mater. 9, 851 (1973).

[9] G.Z. Vinogradova, Glass Formation and Phase Equilibrium in Chalcogenide Systems, Nauka, Moscow 1984.

[10] J.S. Sanghera, J. Heo, J.D. Mackenzie, J. Non-Cryst. Solids 103, 155 (1988).

[11] M. Kępińska, A. Starczewska, P. Duka, M. Nowak, P. Szperlich, Acta Phys. Pol. A 126, 1115 (2014). 